\title{
Classically conditioned enhancement of antibody production
}

\author{
PETER E. JENKINS \\ Vassar College, Poughkeepsie, New York \\ ROBIN A. CHADWICK \\ Yale University, New Haven, Connecticut \\ and \\ JOHN A. NEVIN \\ University of New Hampshire, New Hampshire
}

\begin{abstract}
In Experiments 1 and 2, rats were exposed to a classical conditioning procedure in which saccharin and lithium chloride $(\mathrm{LiCl})$ were paired with an injection of antigen. Relative to control groups, the conditioned animals that received additional saccharin and $\mathrm{LiCl}$ presentations showed significantly higher antibody levels. These experiments demonstrate that classical conditioning procedures are sufficient to establish conditioned reflexes in which stimuli (which were previously neutral with respect to the immune system) enhance antibody production.
\end{abstract}

A number of investigators have demonstrated that antibody production can be suppressed with classical (Pavlovian) conditioning procedures (Ader \& Cohen, 1975; Rogers, Reich, Strom, \& Carpenter, 1976; Wayner, Flannery, \& Singer, 1978). Ader and Cohen (1975) exposed rats to a novel drinking solution (saccharin) that was followed closely in time by an immunosuppressive agent (cyclophosphamide). After this conditioning experience, all rats were injected with antigen. Later exposure of the experimental group to saccharin reduced antibody levels relative to the levels in the control groups. This result demonstrates that a single pairing of saccharin (the conditioned stimulus, CS) with cyclophosphamide (the unconditioned stimulus, US) is sufficient to establish saccharin as a conditioned suppressor of antibody production. The present experiments demonstrate that conditioned enhancement of antibody production is produced by related classical conditioning procedures.

Saccharin (a distinctive taste) and lithium chloride ( $\mathrm{LiCl}$, an elicitor of gastrointestinal upset) were used as the CS because the relationship between the type of CS

Experiment 1 was conducted while the first author was a Postdoctoral Fellow in the Psychology Department of the University of Auckland, Auckland, New Zealand. Experiment 2 was conducted in the laboratories of John A. Nevin and Thomas G. Pistole, with partial support from NSF Grant BNS 79-24082, NSF Grant PCM 8022074, and the University of New Hampshire. We thank Eric Brandin, David Bree, Mary Bullivant, Roger Hill, John Marbrook, Thomas Miller, John Milkens, Thomas G. Pistole, John Tull, and Don Webster for assistance in conducting these experiments. Reprints are available from the first author at Box 249, Vassar College, Poughkeepsie, New York 12601. and the type of US is an important determiner of the magnitude of the conditioning effect (Garcia \& Koelling, 1966). Classical conditioning effects are greatest when the pairing involves a CS and a US that are likely to be correlated in the natural environment (Rozin \& Kalat, 1971). In the rat's natural environment, the consumption of a novel-tasting substance and subsequent gastrointestinal disturbances are events likely to accompany the introduction of an antigen.

\section{METHOD}

In Experiment 1, individually caged 1-year-old male hooded rats were maintained under a 12-h light-dark cycle with ad-lib food. Prior to conditioning, all animals were entrained to consume their water during a $30-\mathrm{min}$ period each morning. This regimen was continued throughout the experiment. Table 1 presents the experimental procedure. The animals were randomly assigned to the conditioned (CS2 and CS0) and nonconditioned (NC) groups. On Day 0, Groups CS2 and CS0 were first presented with a $.1 \%$ saccharin solution for $30 \mathrm{~min}$ instead of tap water. They then received an ip injection of $\mathrm{LiCl}(128 \mathrm{mg} / \mathrm{kg}$ in a volume of $20 \mathrm{ml} / \mathrm{kg}$ ). These manipulations constitute the conditioned stimulus (CS). Next, the rats were exposed to the unconditioned stimulus (US), an ip injection of antigen $(2 \mathrm{ml} / \mathrm{kg}$ of a $1 \%$ thrice-washed suspension of sheep red blood cells). All these manipulations took place within $1 \mathrm{~h}$.

Group NC received the antigen following the daily access to water. On Days 7 and 9, Groups CS2 and NC were exposed to saccharin followed by $\mathrm{LiCl}$. On Day 13, blood was taken from all rats for a hemagglutinating antibody analysis. On Day 16, Groups CS2 and NC were again exposed to the CS, and on Day 20, blood was taken from all rats. Experiment 2 was a replication of Experiment 1. Charles River CD male rats approximately 3 months old were used in Experiment 2, and the experiment was terminated on Day 13. Experiment 2 also included a US group that was conditioned on Day 0 and received additional exposures to the antigen on Days 7 and 9. 
Table 1

Experimental Procedures

\begin{tabular}{|c|c|c|c|c|c|c|c|}
\hline \multirow[b]{2}{*}{ Group } & \multicolumn{2}{|c|}{$\mathrm{N}$} & \multirow[b]{2}{*}{ Day 0} & \multirow[b]{2}{*}{ Days 7 and 9} & \multirow[b]{2}{*}{ Day 13} & \multirow[b]{2}{*}{ Day 16} & \multirow[b]{2}{*}{ Day 20} \\
\hline & $\operatorname{Exp} 1$ & $\operatorname{Exp} 2$ & & & & & \\
\hline $\mathrm{CS} 2$ & 7 & 12 & Sac-LiCl-Ag & Sac-LiCl & $\mathrm{Ab} ?$ & Sac-LiCl & $\mathrm{Ab} ?$ \\
\hline CSO & 7 & 12 & Sac-LiCl-Ag & Tap $\mathrm{H}_{2} \mathrm{O}$ & $\mathrm{Ab}$ ? & $\mathrm{Tap} \mathrm{H}_{2} \mathrm{O}$ & $\mathrm{Ab}$ ? \\
\hline $\mathrm{NC}$ & 6 & 11 & Tap $\mathrm{H}_{2} \mathrm{O}-\mathrm{Ag}$ & $\mathrm{Sac}-\mathrm{LiCl}$ & $\mathrm{Ab}$ ? & $\mathrm{Sac}-\mathrm{LiCl}$ & $\mathrm{Ab}$ ? \\
\hline US & 0 & 12 & Sac-LiCl-Ag & Tap $\mathrm{H}_{2} \mathrm{O}-\mathrm{Ag}$ & $\mathrm{Ab}$ ? & Experime & 2 Terminated \\
\hline
\end{tabular}

Note-Sac $=30-\min$ access to $.1 \%$ saccharin solution; $\mathrm{Ag}=$ ip injection of antigen; $\mathrm{Ab} ?=$ blood taken for antibody analysis.

\section{RESULTS AND DISCUSSION}

If the pairing of the CS and US on Day 0 was sufficient to establish conditioned immunoenhancement, then the antibody titers for Group CS2 should be higher than those for Groups CSO and NC. Group CSO controls for the effects of having the CS and US paired on Day 0, without subsequent CS exposure. Group NC controls for the effects of CS exposure on Days 7 and 9 , without prior CS-US pairing. Group US establishes the effect one would expect if the CS had as great an effect on antibody production as the US (antigen).

Figure 1 presents the results of Experiments 1 and 2. The hemagglutinating antibody analysis of each rat's serum was done in triplicate. If the range of the three titers was greater than 2 or if a rat's mean titer was below 4 (suggesting that the antigen injection was ineffective), then the rat's data were not used in the presentation of the results. This reduced the total $\mathrm{N}$ from 19 to 16 in Experiment 1 and from 47 to 35 in Experiment 2. The reader of the microtiter trays was not aware of the group to which a rat belonged.

In both experiments, Groups NC and CSO were not significantly different from each other on Day 13 (Experiment $1, \mathrm{t}=.85, \mathrm{p}=.418 ;$ Experiment $2, \mathrm{t}=.27$, $\mathrm{p}=.792){ }^{1}$ Consequently, the data from Groups NC and CSO were pooled to form a composite control group

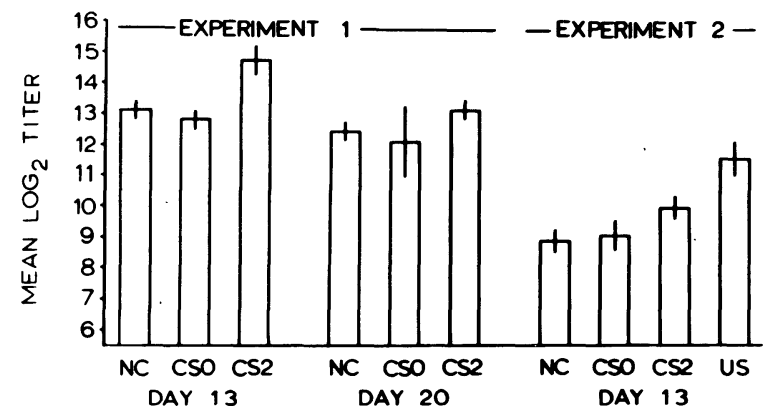

Figure 1. Hemagglutination titers (mean \pm SE) on Days 13 and 20 (Experiment 1) and Day 13 (Experiment 2). On Day 0, Groups CS0, CS2, and US received saccharin and $\mathrm{LiCl}$ paired with antigen. Group NC received tap water and antigen. On Days 7 and 9, Groups NC and CS2 received saccharin and $\mathrm{LiCl}$, Group CS0 received tap water, and Group US received additional antigen. against which to measure the higher predicted titers of Group CS2. In both experiments, the titers of Group CS2 were significantly higher than those of the composite control group (Experiment 1, $\mathrm{t}=4.19, \mathrm{p}=.0012$; Experiment $2, \mathrm{t}=1.84, \mathrm{p}=.043){ }^{2}$ The titers on Day 20 in Experiment 1 are also presented in Figure 1. The data from Groups NC and CSO were again pooled because they were not significantly different from each other $(\mathrm{t}=.329, \mathrm{p}=.759) .^{1}$ Although the difference between Group CS2 and the composite control group was in the predicted direction, it was not statistically significant $(\mathrm{t}=1.404, \mathrm{p}=.094) .^{2}$

The results of Experiments 1 and 2 indicate that the pairing of the CS with the antigen established increased antibody production to the CS. The magnitude of the conditioning effect (i.e., the difference between the $\log _{2}$ titers of Group CS2 and the composite control group) was not large. However, the unconditioned effect (i.e., the difference between the $\log _{2}$ titers of Group US and the composite control group) was also relatively small.

The absolute levels of the titers in Experiment 1 were higher than the titers in Experiment 2. The differences between the rats (age and strain) used in the two experiments and the type of microtiter tray (U-shaped in Experiment 1 and V-shaped in Experiment 2) may have contributed to the differences between the absolute levels of the titers in the two experiments.

It is not likely that the higher titers for Group CS2 were due to the unconditioned effects of the specific $\mathrm{CS}$ (saccharin and $\mathrm{LiCl}$ ) used in these experiments. Comparisons of Group CSO with Group NC reveal that Group NC received a greater number of CS presentations (see Table 1). However, there were no significant differences between Group CSO and Group NC on either Day 13 or Day 20. Ader and Cohen (1975) also showed that $\mathrm{LiCl}$ does not affect antibody levels.

The selective evolutionary forces that might lead to the conditionability of immune responses are easily understood. An organism that can enhance antibody production prior to the introduction of the antigen (i.e., in response to the CS) would clearly have an advantage over an organism that did not enhance antibody production until the antigen (US) was introduced.

The present experiments extend previous research on conditioned immunosuppression by demonstrating 
conditioned enhancement of immune responses in rats. Further analysis of the relation between immune responses and an individual's learning history may aid in understanding and treating many human health problems, including cancer, as well as contribute to the study of immune system mechanisms and conditioning processes.

\section{REFERENCES}

Ader, R., \& Cohen, N. Behaviorally conditioned immunosuppression. Psychosomatic Medicine, 1975, 37, 333-340.

Garcia, J., \& Koelling, R. A. Relation of cue to consequences in avoidance learning. Psychonomic Sciences, 1966, 4, 123-124.

Rogers, M. P., Reich, P., Strom, T. B., \& Carpenter, C. B.
Behaviorally conditioned immunosuppression: Replication of a recent study. Psychosomatic Medicine, 1976, 38, 447-451.

Rozin, P., \& Kalat, J. W. Specific hungers and poisoning as adaptive specializations of learning. Psychological Review, 1971, 78, 459-486.

Wayner, E. A., Flannery, G. R., \& Singer, G. Effects of taste aversion conditioning on the primary antibody response to sheep red blood cells and Brucella abortus in the albino rat. Physiology \& Behavior, 1978, 21, 995-1000.

\section{NOTES}

1. These significance levels are based on two-tailed probabilities.

2. These significance levels are based on one-tailed probabilities.

(Manuscript received for publication May 20, 1983.) 